\title{
Subtleties in the BABAR measurement of time-reversal violation
}

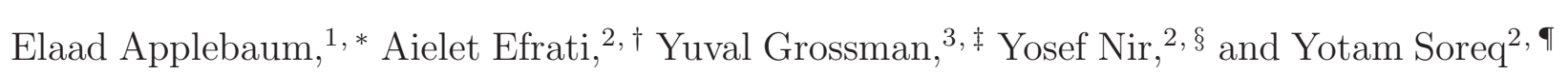 \\ ${ }^{1}$ Department of Physics, University of Illinois at \\ Urbana-Champaign, Urbana, Illinois 61801, USA \\ ${ }^{2}$ Department of Particle Physics and Astrophysics \\ Weizmann Institute of Science, Rehovot 7610001, Israel \\ ${ }^{3}$ Laboratory for Elementary-Particle Physics, \\ Cornell University, Ithaca, New York 14853, USA
}

\begin{abstract}
A first measurement of time-reversal $(\mathrm{T}$ ) asymmetries that are not also $\mathrm{CP}$ asymmetries has been recently achieved by the $B A B A R$ collaboration. We analyze the measured asymmetries in the presence of direct $\mathrm{CP}$ violation, CPT violation, wrong strangeness decays and wrong sign semileptonic decays. We note that the commonly used $S_{\psi K}$ and $C_{\psi K}$ parameters are CP-odd, but have a T-odd CPT-even part and a T-even CPT-odd part. We introduce parameters that have well-defined transformation properties under CP, T and CPT. We identify contributions to the measured asymmetries that are $\mathrm{T}$ conserving. We explain why, in order that the measured asymmetries would be purely odd under time-reversal, there is no need to assume the absence of direct CP violation. Instead, one needs to assume (i) the absence of CPT violation in strangeness changing decays, and (ii) the absence of wrong sign decays.
\end{abstract}

\footnotetext{
* eappleb2@illinois.edu

$\dagger$ aielet.efrati@weizmann.ac.il

$\ddagger$ yg73@cornell.edu

$\S$ yosef.nir@weizmann.ac.il

ฯ yotam.soreq@weizmann.ac.il
} 


\section{INTRODUCTION}

The BABAR collaboration has recently announced the first direct observation of timereversal violation in the neutral $B$ meson system [1]. The basic idea is to compare the time-dependent rates of two processes that differ by exchange of initial and final states. The measurement makes use of the Einstein-Podolsky-Rosen (EPR) effect in the entangled $B$ mesons produced in $\Upsilon(4 S)$ decays [2 6] ]. For example, one rate, $\Gamma_{\left(\psi K_{L}\right)_{\perp}, \ell^{-} X}$, involves the decay of one of the neutral $B$ 's into a $\psi K_{L}$ state, and, after time $t$, the decay of the other $B$ into $\ell^{-} X$. The other rate, $\Gamma_{\left(\ell^{+} X\right)_{\perp}, \psi K_{S}}$, involves the decay of one of the neutral $B$ 's into $\ell^{+} X$, and, after time $t$, the decay of the other $B$ into $\psi K_{S}$. Under certain assumptions, to be spelled out below, this is a comparison between the rates of $B_{-} \rightarrow \bar{B}^{0}$ and $\bar{B}^{0} \rightarrow B_{-}$, where $\bar{B}^{0}$ has a well-defined flavor content $(b \bar{d})$ and $B_{-}$is a CP-odd state. The asymmetry is defined as follows:

$$
A_{T} \equiv \frac{\Gamma_{\left(\psi K_{L}\right)_{\perp}, \ell^{-} X}-\Gamma_{\left(\ell^{+} X\right)_{\perp}, \psi K_{S}}}{\Gamma_{\left(\psi K_{L}\right)_{\perp}, \ell^{-} X}+\Gamma_{\left(\ell^{+} X\right)_{\perp}, \psi K_{S}}} .
$$

$B A B A R$ take the time dependence of the asymmetry to be of the form

$$
A_{T}=\frac{\Delta S_{T}^{+}}{2} \sin (\Delta m t)+\frac{\Delta C_{T}^{+}}{2} \cos (\Delta m t) .
$$

(A more accurate description of the BABAR analysis is given in Section III.) They obtain

$$
\Delta S_{T}^{+}=-1.37 \pm 0.15, \quad \Delta C_{T}^{+}=+0.10 \pm 0.16 .
$$

The fact that $\Delta S_{T}^{+} \neq 0$ constitutes their direct demonstration of time reversal violation.

Time reversal violation had been observed earlier in the neutral $K$ meson system by the CPLEAR collaboration [7]. The measurement involves the processes $p \bar{p} \rightarrow K^{-} \pi^{+} K^{0}$ and $p \bar{p} \rightarrow K^{+} \pi^{-} \bar{K}^{0}$. Again, one aims to compare rates of processes that are related by exchange of initial and final states. One rate, $\Gamma_{K^{-}, e^{-}}$, involves a production of $K^{-}$and a neutral $K$ that after time $t$ decay into $e^{-} \pi^{+} \bar{\nu}$. The other rate, $\Gamma_{K^{+}, \ell^{+}}$, involves the production of $K^{+}$and a neutral $\bar{K}$ that after time $t$ decay into $e^{+} \pi^{-} \nu$. Under certain assumptions, this is a comparison between the rates of $K^{0} \rightarrow \bar{K}^{0}$ and $\bar{K}^{0} \rightarrow K^{0}$ [8]. The time dependent asymmetry is defined as follows:

$$
A_{T, K} \equiv \frac{\Gamma_{K^{+}, e^{+}}-\Gamma_{K^{-}, e^{-}}}{\Gamma_{K^{+}, e^{+}}+\Gamma_{K^{-}, e^{-}}} .
$$

CPLEAR integrate the rates over times $\tau_{S} \leq t \leq 20 \tau_{S}\left(\tau_{S}\right.$ is the lifetime of $\left.K_{S}\right)$, and obtain

$$
\left\langle A_{T, K}\right\rangle_{(1-20) \tau_{S}}=(6.6 \pm 1.6) \times 10^{-3} .
$$

The CPLEAR asymmetry is also a CP asymmetry, since the initial and final states are $\mathrm{CP}$-conjugate. In contrast, the BABAR asymmetry is not a $\mathrm{CP}$ asymmetry.

In this work, we examine the analysis of $A_{T}$, with the aim of answering the following two related questions: 
- Would it vanish in the T-symmetry limit?

- Is the initial state in each of the two processes the T-conjugate of the final state in the other?

To answer these questions, it is helpful to use only parameters that have well-defined transformation properties under all three relevant symmetries: CP, T and CPT. In most of the related literature, $\mathrm{CPT}$ conservation is assumed, and then parameters that are $\mathrm{CP}$-odd or CP-even are used. For example, the parameters $\Delta S_{T}^{+}$and $\Delta C_{T}^{+}$of Eq. (21) are both CP-odd. However, when aiming to demonstrate that time-reversal is violated, one needs to allow for $\mathrm{CPT}$ violation [9, 10]. (Otherwise, $\mathrm{T}$ violation follows from $\mathrm{CP}$ violation.) In this case, most of the parameters used in the literature do not have well-defined transformation under $\mathrm{T}$ and CPT. In particular, $\Delta S_{T}^{+}$and $\Delta C_{T}^{+}$have, apart from a T-odd CPT-even part, also a T-even CPT-odd part.

In Section \we present our formalism and, in particular, introduce parameters with welldefined transformation properties under CP, T and CPT. In Section $\amalg$ we derive expressions for the asymmetries measured by BABAR in terms of these parameters. The results allow us to answer the first question and to clearly formulate the assumptions one needs to make in order to identify the asymmetries measured by BABAR with time reversal violation. In Section [IIC we comment on the time-dependence of the asymmetry under various approximations. As concerns the second question, for two processes to be related by time-reversal, the initial state in each of them should be the time-reversal conjugate of the final state in the other. The subtleties related to this requirement, in the context of the BABAR measurements, are clarified in Section IV. We conclude in Section D. In Appendix A we compile relevant experimental constraints on $\mathrm{CP}$ violation in mixing and in decay, on CPT violation, and on wrong-strangeness and wrong-sign $B$ decays. In Appendix $B$ we present combinations of measurements that allow us to isolate various parameters of interest. Appendix [C contains the full expressions for all the asymmetries measured at BABAR. In Appendix $D$ we define "theoretical asymmetries" (or, equivalently, gedanken experiments) involving inverse decays, which provide another way to shed light on the subtleties of experimentally demonstrating time reversal violation. In Appendix E we show how CPT violation affects the EPR entanglement of the initial $B$ meson state.

\section{DEFINITIONS AND NOTATIONS}

We use a formalism that allows for CPT violation. Similar to Ref. [11], we use in and out states defined by the reciprocal basis [12]. Translating our notations to those of Ref. [13] is straightforward. 


\section{A. $B^{0}-\bar{B}^{0}$ mixing}

The effective Hamiltonian $H=M-i \Gamma / 2$ describing the evolution of the oscillating system is non-Hermitian. It is thus diagonalized by a general similarity transformation,

$$
X^{-1} H X=\operatorname{diag}\left(\omega_{H}, \omega_{L}\right)
$$

where

$$
X=\left(\begin{array}{cc}
p \sqrt{1+z} & p \sqrt{1-z} \\
-q \sqrt{1-z} & q \sqrt{1+z}
\end{array}\right)
$$

with $|p|^{2}+|q|^{2}=1$. The complex parameter $z \equiv z^{R}+i z^{I}$ represents CP and CPT violation in mixing. We define a real parameter, $R_{M}$, representing $\mathrm{T}$ and $\mathrm{CP}$ violation in mixing:

$$
R_{M} \equiv \frac{1-|q / p|^{2}}{1+|q / p|^{2}} .
$$

In Eq. (77) we omitted normalization factors that deviate from unity only in $\mathcal{O}\left(R_{M} z, z^{2}\right)$.

For the mass and width eigenvalues, we use

$$
\begin{aligned}
& \omega_{H, L}=m_{H, L}-\frac{i}{2} \Gamma_{H, L}, \quad m=\frac{m_{L}+m_{H}}{2}, \quad \Gamma=\frac{\Gamma_{L}+\Gamma_{H}}{2}, \\
& x=\frac{m_{H}-m_{L}}{\Gamma}, \quad y=\frac{\Gamma_{H}-\Gamma_{L}}{2 \Gamma} .
\end{aligned}
$$

The incoming mass eigenstates are

$$
\begin{aligned}
\left|B_{H}^{\mathrm{in}}\right\rangle & =p \sqrt{1+z}\left|B^{0}\right\rangle-q \sqrt{1-z}\left|\bar{B}^{0}\right\rangle \\
\left|B_{L}^{\mathrm{in}}\right\rangle & =p \sqrt{1-z}\left|B^{0}\right\rangle+q \sqrt{1+z}\left|\bar{B}^{0}\right\rangle .
\end{aligned}
$$

Their time evolution is given by

$$
\left|B_{H, L}^{\mathrm{in}}(t)\right\rangle=e^{-i \omega_{H, L} t}\left|B_{H, L}^{\mathrm{in}}\right\rangle .
$$

The outgoing states are

$$
\begin{aligned}
& \left\langle B_{H}^{\text {out }}\right|=\frac{1}{2 p q}\left(q \sqrt{1+z}\left\langle B^{0}\right|-p \sqrt{1-z}\left\langle\overline{B^{0}}\right|\right), \\
& \left\langle B_{L}^{\text {out }}\right|=\frac{1}{2 p q}\left(q \sqrt{1-z}\left\langle B^{0}\right|+p \sqrt{1+z}\left\langle\bar{B}^{0}\right|\right) .
\end{aligned}
$$

\section{B. $B$ decay amplitudes}

We define decay amplitudes,

$$
A_{f} \equiv A\left(B^{0} \rightarrow f\right)=\left\langle f|T| B^{0}\right\rangle, \quad \bar{A}_{f} \equiv A\left(\bar{B}^{0} \rightarrow f\right)=\left\langle f|T| \bar{B}^{0}\right\rangle
$$


and the inverse-decay amplitudes,

$$
A_{f}^{\mathrm{ID}} \equiv A\left(f^{T} \rightarrow B^{0}\right)=\left\langle B^{0}|T| f^{T}\right\rangle, \quad \bar{A}_{f}^{\mathrm{ID}} \equiv A\left(f^{T} \rightarrow \bar{B}^{0}\right)=\left\langle\bar{B}^{0}|T| f^{T}\right\rangle,
$$

where $f^{T}$ is T-conjugate (i.e. reversed spins and momenta) to $f$. CP violation in decay and in inverse decay is manifest in the direct asymmetries

$$
\mathcal{A}_{f} \equiv \frac{\left|\bar{A}_{\bar{f}}\right|^{2}-\left|A_{f}\right|^{2}}{\left|\bar{A}_{\bar{f}}\right|^{2}+\left|A_{f}\right|^{2}}, \quad \mathcal{A}_{f}^{\mathrm{ID}} \equiv \frac{\left|A_{f}^{\mathrm{ID}}\right|^{2}-\left|\bar{A}_{\bar{f}}^{\mathrm{ID}}\right|^{2}}{\left|A_{f}^{\mathrm{ID}}\right|^{2}+\left|\bar{A}_{\bar{f}}^{\mathrm{ID}}\right|^{2}},
$$

where $\bar{f}$ is CP-conjugate to $f$.

We define complex parameters, $\theta_{f}$, representing CPT violation in the decay:

$$
\theta_{f}=\theta_{f}^{R}+i \theta_{f}^{I} \equiv \frac{A_{\bar{f}}^{\mathrm{ID}} / \bar{A}_{\bar{f}}^{\mathrm{ID}}-\bar{A}_{f} / A_{f}}{A_{\bar{f}}^{\mathrm{ID}} / \bar{A}_{\bar{f}}^{\mathrm{ID}}+\bar{A}_{f} / A_{f}} .
$$

Under CP, $\theta_{f} \rightarrow-\theta_{\bar{f}}$, while under T, $\theta_{f} \rightarrow \theta_{\bar{f}}$. Thus, for final CP eigenstates, $\theta_{f} \neq 0$ breaks CPT and CP, but not T.

We further define the phase convention independent combination of amplitudes and mixing parameters,

$$
\lambda_{f} \equiv \frac{q}{p} \frac{\bar{A}_{f}}{A_{f}} \sqrt{\frac{1+\theta_{f}}{1-\theta_{f}}}=\frac{q}{p} \frac{A_{\bar{f}}^{\mathrm{ID}}}{\bar{A}_{\bar{f}}^{\mathrm{ID}}} \sqrt{\frac{1-\theta_{f}}{1+\theta_{f}}} .
$$

In the CPT limit $z=\theta_{f}=0$ and the standard definition of $\lambda_{f}$ is recovered. It is convenient to introduce the following functions of $\lambda_{f}$ :

$$
C_{f} \equiv \frac{1-\left|\lambda_{f}\right|^{2}}{1+\left|\lambda_{f}\right|^{2}}, \quad S_{f} \equiv \frac{2 \mathcal{I} m\left(\lambda_{f}\right)}{1+\left|\lambda_{f}\right|^{2}}, \quad G_{f} \equiv \frac{2 \mathcal{R} e\left(\lambda_{f}\right)}{1+\left|\lambda_{f}\right|^{2}}
$$

with $C_{f}^{2}+G_{f}^{2}+S_{f}^{2}=1$.

We emphasize that the definition of $\lambda_{f}$ in Eq. (17) and, consequently, the definitions of $S_{f}, C_{f}$ and $G_{f}$ in Eq. (18) differ from the standard definitions of these parameters in the literature. Our definition lends itself straightforwardly to the theoretical analysis that we are doing. The standard definition lends itself straightforwardly to the description of the experimentally measured rates. The relation between the two will be further clarified in the next subsection. The distinction between the two is crucial for understanding the subtleties in the interpretation of the BABAR measurements. Of course, in the absence of CPT violation, the two sets of definitions coincide.

\section{T, CP and CPT transformations}

The transformation rules for the parameters defined in the previous subsection under T, $\mathrm{CP}$ and CPT, are summarized in Table \. 


\begin{tabular}{|c|c|c|c|}
\hline \hline Parameter & $\mathrm{T}$ & $\mathrm{CP}$ & $\mathrm{CPT}$ \\
\hline \hline$R_{M}$ & $-R_{M}$ & $-R_{M}$ & $R_{M}$ \\
$z$ & $z$ & $-z$ & $-z$ \\
$\lambda_{f}$ & $1 / \lambda_{\bar{f}}$ & $1 / \lambda_{\bar{f}}$ & $\lambda_{f}$ \\
$S_{f}$ & $-S_{\bar{f}}$ & $-S_{\bar{f}}$ & $S_{f}$ \\
$C_{f}$ & $-C_{\bar{f}}$ & $-C_{\bar{f}}$ & $C_{f}$ \\
$G_{f}$ & $G_{\bar{f}}$ & $G_{\bar{f}}$ & $G_{f}$ \\
$\mathcal{A}_{f}$ & $-\mathcal{A}_{f}^{\mathrm{ID}}$ & $-\mathcal{A}_{f}$ & $\mathcal{A}_{f}^{\mathrm{ID}}$ \\
$\theta_{f}$ & $\theta_{\bar{f}}$ & $-\theta_{\bar{f}}$ & $-\theta_{f}$ \\
\hline \hline
\end{tabular}

TABLE I. Transformation rules of the various parameters under T, CP and CPT

As explained above, it is very convenient for our purposes to use parameters that are either even or odd under all three transformations. Using superscript + for T-even, and for T-odd, we define the following combinations:

$$
\begin{aligned}
C_{f}^{-} & =\frac{1}{2}\left(C_{f}+C_{\bar{f}}\right), & C_{f}^{+} & =\frac{1}{2}\left(C_{f}-C_{\bar{f}}\right), \\
S_{f}^{-} & =\frac{1}{2}\left(S_{f}+S_{\bar{f}}\right), & S_{f}^{+} & =\frac{1}{2}\left(S_{f}-S_{\bar{f}}\right), \\
G_{f}^{-} & =\frac{1}{2}\left(G_{f}-G_{\bar{f}}\right), & G_{f}^{+} & =\frac{1}{2}\left(G_{f}+G_{\bar{f}}\right), \\
\theta_{f}^{-} & =\frac{1}{2}\left(\theta_{f}-\theta_{\bar{f}}\right), & \theta_{f}^{+} & =\frac{1}{2}\left(\theta_{f}+\theta_{\bar{f}}\right) .
\end{aligned}
$$

We further define

$$
\mathcal{A}_{f}^{-}=\frac{\mathcal{A}_{f}+\mathcal{A}_{f}^{\mathrm{ID}}}{2}=R_{M}-C_{f_{C P}}, \quad \mathcal{A}_{f}^{+}=\frac{\mathcal{A}_{f}-\mathcal{A}_{f}^{\mathrm{ID}}}{2}=-\theta_{f_{C P}^{+, R}},
$$

where the last step in each equation is relevant only for CP eigenstates and we expand to linear order in $C_{f_{C P}}, R_{M}$ and $\theta_{f_{C P}}$. A summary of the transformation properties of these parameters is provided in Table II. For final CP eigenstates, such as $f=\psi K_{S, L}, S_{f}$ and $C_{f}$ are T-odd, while $G_{f}$ and $\theta_{f}$ are T-even, so that $S_{f}^{+}=C_{f}^{+}=G_{f}^{-}=\theta_{f}^{-}=0$. We summarize the transformation properties for final CP eigenstates also in Table II.

In practice, inverse decays are not accessible to the experiments. In particular, experiments are not sensitive to $\lambda_{f}$, as defined in Eq. (17), but to the related observable $\lambda_{f}^{e}$, defined via

$$
\lambda_{f}^{e} \equiv \frac{q}{p} \frac{\bar{A}_{f}}{A_{f}}=\lambda_{f}\left(1-\theta_{f}\right),
$$

where the second equation holds to first order in $\theta_{f}$. Accordingly, the experiments are 


\begin{tabular}{|c|c|c|c|}
\hline \hline Parameter & $\mathrm{T}$ & $\mathrm{CP}$ & $\mathrm{CPT}$ \\
\hline \hline$R_{M}, S_{f}^{-}, C_{f}^{-}, G_{f}^{-}, \mathcal{A}_{f}^{-}$ & - & - & + \\
$z, \theta_{f}^{+}, \mathcal{A}_{f}^{+}$ & + & - & - \\
$\theta_{f}^{-}$ & - & + & - \\
$S_{f}^{+}, C_{f}^{+}, G_{f}^{+}$ & + & + & + \\
\hline$S_{f_{C P}, C_{f_{C P}}}$ & - & - & + \\
$\theta_{f_{C P}}$ & + & - & - \\
$G_{f_{C P}}$ & + & + & + \\
\hline \hline
\end{tabular}

TABLE II. Eigenvalues of the various parameters under T, CP and CPT

sensitive to the following parameters:

$$
\begin{aligned}
C_{f}^{e} & =C_{f}+\left(1-C_{f}^{2}\right) \theta_{f}^{R}, \\
S_{f}^{e} & =S_{f}\left(1-C_{f} \theta_{f}^{R}\right)-G_{f} \theta_{f}^{I}, \\
G_{f}^{e} & =G_{f}\left(1-C_{f} \theta_{f}^{R}\right)+S_{f} \theta_{f}^{I} .
\end{aligned}
$$

\section{Wrong-strangeness and wrong-sign decays}

Among the final CP eigenstates, we focus on decays into the final $\psi K_{S, L}$ states (neglecting effects of $\left.\epsilon_{K}\right)$. We distinguish between the right strangeness decays,

$$
A_{K^{0}}=A\left(B^{0} \rightarrow K^{0}\right), \quad \bar{A}_{\bar{K}^{0}}=A\left(\bar{B}^{0} \rightarrow \bar{K}^{0}\right),
$$

and the wrong strangeness decays,

$$
\bar{A}_{K^{0}}=A\left(\bar{B}^{0} \rightarrow K^{0}\right), \quad A_{\bar{K}^{0}}=A\left(B^{0} \rightarrow \bar{K}^{0}\right) .
$$

We define

$$
\begin{aligned}
\hat{C}_{\psi K} & \equiv \frac{1}{2}\left(C_{\psi K_{S}}+C_{\psi K_{L}}\right), & \Delta C_{\psi K} & \equiv \frac{1}{2}\left(C_{\psi K_{S}}-C_{\psi K_{L}}\right), \\
\hat{S}_{\psi K} & \equiv \frac{1}{2}\left(S_{\psi K_{S}}-S_{\psi K_{L}}\right), & \Delta S_{\psi K} & \equiv \frac{1}{2}\left(S_{\psi K_{S}}+S_{\psi K_{L}}\right), \\
\hat{G}_{\psi K} & \equiv \frac{1}{2}\left(G_{\psi K_{S}}-G_{\psi K_{L}}\right), & \Delta G_{\psi K} & \equiv \frac{1}{2}\left(G_{\psi K_{S}}+G_{\psi K_{L}}\right),
\end{aligned}
$$

and

$$
\hat{\theta}_{\psi K} \equiv \frac{1}{2}\left(\theta_{\psi K_{L}}+\theta_{\psi K_{S}}\right), \quad \Delta \theta_{\psi K} \equiv \frac{1}{2}\left(\theta_{\psi K_{S}}-\theta_{\psi K_{L}}\right) .
$$

In the limit of no wrong strangeness decay, $\lambda_{\psi K_{S}}=-\lambda_{\psi K_{L}}$ [14] (Ref. [14] assumes CPT conservation, but this is not a necessary assumption) and, consequently, $\Delta C_{\psi K}, \Delta G_{\psi K}, \Delta S_{\psi K}$ and $\Delta \theta_{\psi K}$ vanish. 
Among the flavor specific final states, we focus on decays into final $\ell^{ \pm} X$ states. Here we distinguish between the right sign decays,

$$
A_{\ell^{+}}=A\left(B^{0} \rightarrow \ell^{+} X\right), \quad \bar{A}_{\ell^{-}}=A\left(\bar{B}^{0} \rightarrow \ell^{-} X\right)
$$

and the wrong sign decays,

$$
A_{\ell^{-}}=A\left(B^{0} \rightarrow \ell^{-} X\right), \quad \bar{A}_{\ell^{+}}=A\left(\bar{B}^{0} \rightarrow \ell^{+} X\right)
$$

We define $C_{\ell}^{ \pm}, S_{\ell}^{ \pm}$and $G_{\ell}^{ \pm}$according to Eq. (19), with $f=\ell^{+}$, and a super-index $+(-)$ denoting a $\mathrm{T}$ conserving (violating) combination. Taking the wrong sign decays to be much smaller in magnitude than the right sign decays, we have $\left|\lambda_{\ell^{+}}\right| \ll 1$ and $\left|\lambda_{\ell^{-}}^{-1}\right| \ll 1$. We will work to first order in $\left|\lambda_{\ell^{+}}\right|$and in $\left|\lambda_{\ell^{-}}^{-1}\right|$, which means that we set $C_{\ell}^{+}=1$ and $C_{\ell}^{-}=0$. On the other hand, the four other relevant parameters are linear in $\left|\lambda_{\ell^{+}}\right|$and in $\left|\lambda_{\ell^{-}}^{-1}\right|$ :

$$
S_{\ell}^{ \pm} \simeq \mathcal{I} m\left(\lambda_{\ell^{+}} \pm \lambda_{\ell^{-}}^{-1}\right), \quad G_{\ell}^{ \pm} \simeq \mathcal{R} e\left(\lambda_{\ell^{+}} \pm \lambda_{\ell^{-}}^{-1}\right)
$$

\section{TIME-REVERSAL ASYMMETRIES}

\section{A. The master formula}

Consider a pair of $B$-mesons produced in $\Upsilon(4 S)$ decay, where one of the $B$-mesons decays at time $t_{1}$ to a final state $f_{1}$, and the other $B$-meson decays at a later time, $t_{2}=t_{1}+t$, to a final state $f_{2}$. The time dependent rate for this process, to linear order in $R_{M}, z$ and $\theta$, is given by

$$
\begin{aligned}
\Gamma_{\left(f_{1}\right)_{\perp}, f_{2}} & =N_{(1)_{\perp}, 2} e^{-\Gamma\left(t_{1}+t_{2}\right)} \\
& \times\left[\kappa_{(1)_{\perp}, 2} \cosh (y \Gamma t)+\sigma_{(1)_{\perp}, 2} \sinh (y \Gamma t)+C_{(1)_{\perp}, 2} \cos (x \Gamma t)+S_{(1)_{\perp}, 2} \sin (x \Gamma t)\right] .
\end{aligned}
$$


where

$$
\begin{aligned}
& N_{(1)_{\perp}, 2}=\left[1+\left(C_{1}+C_{2}\right)\left(R_{M}-z^{R}\right)\right] \mathcal{N}_{1} \mathcal{N}_{2}, \\
& \kappa_{(1)_{\perp}, 2}=\left[1-G_{1} G_{2}+\left(C_{1}+C_{2}+C_{2} G_{1}+C_{1} G_{2}-C_{2} G_{2} G_{1}-C_{1} G_{2} G_{1}\right) z^{R}\right. \\
& \left.-\left(S_{1}+S_{2}\right) z^{I}+G_{1} G_{2}\left(C_{2} \theta_{2}^{R}+C_{1} \theta_{1}^{R}\right)-G_{1} S_{2} \theta_{2}^{I}-G_{2} S_{1} \theta_{1}^{I}\right], \\
& \sigma_{(1) \perp, 2}=\left[G_{2}-G_{1}-\left(C_{2}-C_{1}-C_{2} G_{2}-C_{1} G_{2}+C_{2} G_{1}+C_{1} G_{1}\right) z^{R}\right. \\
& \left.-\left(G_{2} S_{1}-G_{1} S_{2}\right) z^{I}-C_{2} G_{2} \theta_{2}^{R}+S_{2} \theta_{2}^{I}+C_{1} G_{1} \theta_{1}^{R}-S_{1} \theta_{1}^{I}\right], \\
& C_{(1)_{\perp}, 2}=-\left[C_{2} C_{1}+S_{2} S_{1}+\left(C_{2}^{2} C_{1}+C_{2} C_{1}^{2}+C_{1} G_{2}+C_{2} G_{1}+C_{2} S_{2} S_{1}+C_{1} S_{2} S_{1}\right) z^{R}\right. \\
& -\left(S_{2}+S_{1}\right) z^{I}-G_{2} S_{1} \theta_{2}^{I}+\left(C_{1}-C_{2}^{2} C_{1}-C_{2} S_{2} S_{1}\right) \theta_{2}^{R} \\
& \left.-G_{1} S_{2} \theta_{1}^{I}+\left(C_{2}-C_{2} C_{1}^{2}-C_{1} S_{2} S_{1}\right) \theta_{1}^{R}\right] \text {, } \\
& S_{(1)_{\perp}, 2}=\left[C_{1} S_{2}-C_{2} S_{1}+\left(C_{2} C_{1} S_{2}+C_{1}^{2} S_{2}+G_{1} S_{2}-C_{2}^{2} S_{1}-C_{2} C_{1} S_{1}-G_{2} S_{1}\right) z^{R}\right. \\
& +\left(C_{2}-C_{1}\right) z^{I}-C_{1} G_{2} \theta_{2}^{I}+\left(C_{2}^{2} S_{1}-C_{2} C_{1} S_{2}-S_{1}\right) \theta_{2}^{R} \\
& \left.+C_{2} G_{1} \theta_{1}^{I}-\left(C_{1}^{2} S_{2}-C_{2} C_{1} S_{1}-S_{2}\right) \theta_{1}^{R}\right] \text {, }
\end{aligned}
$$

and, for the sake of brevity, we denote $X_{i} \equiv X_{f_{i}}$ for $X=S, C, G, \theta$ and $\mathcal{N}_{i}=\left(\left|A_{f_{i}}\right|^{2}+\left|\bar{A}_{f_{i}}\right|^{2}\right)$. Eq. (30) is our "master formula", and serves as the starting point for all our calculations. Note that the decomposition into $e^{-\Gamma\left(t_{1}+t_{2}\right)} \times f(t)$ for the $\Upsilon(4 S)$ decay products holds even in the presence of CPT violation. (See Appendix E.)

\section{B. The BABAR T-asymmetry}

In what follows we set $y=0, C_{\ell}^{+}=1, C_{\ell}^{-}=0$ and $\epsilon_{K}=0$. We do so for the sake of simplicity: All these effects can, in principle, be accounted for. (Care is needed when accounting for the effect of Kaon mixing [15 20].) We work to linear order in the following small parameters:

$$
R_{M}, z^{R}, z^{I}, \theta_{f}^{R}, \theta_{f}^{I}, \hat{C}_{\psi K}, \Delta C_{\psi K}, \Delta S_{\psi K}, \Delta G_{\psi K}, S_{\ell}^{ \pm}, G_{\ell}^{ \pm}
$$

We consider the two processes that are relevant to the experimentally measured asymmetry (1). Using the master formulas (30), and implementing our approximations, we obtain 
the following time-dependent decay rates:

$$
\begin{aligned}
\Gamma_{\left(\psi K_{L}\right)_{\perp}, \ell^{-} X} & =\left[1-R_{M}+z^{R}\right] \mathcal{N}_{\psi K_{L}} \mathcal{N}_{\ell^{-} X} \\
& \times\left\{\left[1-G_{\psi K_{L}} G_{\ell^{-}}-\left(1+G_{\psi K_{L}}\right) z^{R}-S_{\psi K_{L}} z^{I}\right]\right. \\
& -\left[-C_{\psi K_{L}}+S_{\ell^{-}} S_{\psi K_{L}}-G_{\psi K_{L}} z^{R}-S_{\psi K_{L}} z^{I}-\theta_{\psi K_{L}}^{R}\right] \cos (x \Gamma t) \\
& \left.+\left[S_{\psi K_{L}}-S_{\psi K_{L}} z^{R}-z^{I}-G_{\psi K_{L}} \theta_{\psi K_{L}}^{I}\right] \sin (x \Gamma t)\right\}, \\
\Gamma_{\left(\ell^{+} X\right)_{\perp}, \psi K_{S}} & =\left[1+R_{M}-z^{R}\right] \mathcal{N}_{\ell^{+} X} \mathcal{N}_{\psi K_{S}} \\
& \times\left\{\left[1-G_{\ell^{+}} G_{\psi K_{S}}+\left(1+G_{\psi K_{S}}\right) z^{R}-S_{\psi K_{S}} z^{I}\right]\right. \\
& -\left[C_{\psi K_{S}}+S_{\psi K_{S}} S_{\ell^{+}}+G_{\psi K_{S}} z^{R}-S_{\psi K_{S}} z^{I}+\theta_{\psi K_{S}}^{R}\right] \cos (x \Gamma t) \\
& \left.+\left[S_{\psi K_{S}}+S_{\psi K_{S}} z^{R}-z^{I}-G_{\psi K_{S}} \theta_{\psi K_{S}}^{I}\right] \sin (x \Gamma t)\right\}
\end{aligned}
$$

where the overall decay exponential factor is omitted. The analysis performed by BABAR, as described in Ref. [1], is as follows. The time dependent decay rates are measured and fitted to time-dependence of the form (30), approximating (as we do) $y=0$. The quantities $\Delta S_{T}^{+}$and $\Delta C_{T}^{+}$, to which the $B A B A R$ results of (3) apply, correspond to the following combinations:

$$
\begin{aligned}
& \Delta S_{T}^{+}=\frac{S_{\left(\psi K_{L}\right)_{\perp}, \ell^{-} X}}{\kappa_{\left(\psi K_{L}\right)_{\perp}, \ell^{-} X}}-\frac{S_{\left(\ell^{+} X\right)_{\perp}, \psi K_{S}}}{\kappa_{\left(\ell^{+} X\right)_{\perp}, \psi K_{S}}} \equiv S_{\ell^{-}, K_{L}}^{-}-S_{\ell^{+}, K_{S}}^{+}, \\
& \Delta C_{T}^{+}=\frac{C_{\left(\psi K_{L}\right)_{\perp}, \ell^{-} X}}{\kappa_{\left(\psi K_{L}\right)_{\perp}, \ell^{-} X}}-\frac{C_{\left(\ell^{+} X\right)_{\perp}, \psi K_{S}}}{\kappa_{\left(\ell^{+} X\right)_{\perp}, \psi K_{S}}} \equiv C_{\ell^{-}, K_{L}}^{-}-C_{\ell^{+}, K_{S}}^{+} .
\end{aligned}
$$

The last identity relates our definitions in Eq. (30) with those of BABAR in Ref. [1]. In the latter, a super-index + refers to the case the leptonic tag occurs before the CP tag, as in $\Gamma_{\left(\ell^{+} X\right)_{\perp}, \psi K_{S}}$, while a super-index - refers to the case that the CP tag occurs before the leptonic tag, as in $\Gamma_{\left(\psi K_{L}\right)_{\perp}, \ell^{-} X}$. We note that the normalization of Eqs. (35) removes the dependence on the total production rates and effects such as direct $\mathrm{CP}$ violation in leptonic decays.

We obtain the following expressions for these observables:

$$
\begin{aligned}
\Delta S_{T}^{+} & =-2\left[\left(\hat{S}_{\psi K}-\hat{G}_{\psi K} \hat{\theta}_{\psi K}^{I}\right)+\hat{S}_{\psi K} \hat{G}_{\psi K}\left(G_{\ell}^{-}-z^{R}\right)\right], \\
\Delta C_{T}^{+} & =2\left[\left(\hat{C}_{\psi K}+\hat{\theta}_{\psi K}^{R}\right)+\hat{S}_{\psi K}\left(S_{\ell}^{-}-z^{I}\right)\right] .
\end{aligned}
$$

If we switch off all the T-odd parameters, we are left with the following $\mathrm{T}$ conserving (TC) contributions:

$$
\begin{aligned}
& \Delta S_{T}^{+}(\text {T-odd parameters }=0)=2 \hat{G}_{\psi K} \hat{\theta}_{\psi K}^{I}, \\
& \Delta C_{T}^{+}(\text {T-odd parameters }=0)=2 \hat{\theta}_{\psi K}^{R} .
\end{aligned}
$$


These contributions are CPT violating. Yet, since $\hat{\theta}_{\psi_{K}}$ involves inverse decays, we are not aware of any way to experimentally bound it, and to exclude the possibility that it generates the measured value of $\Delta S_{T}^{+}$. We would like to emphasize, however, the following three points.

- The appearance of the T conserving, CPT violating effects should come as no surprise. As explained in the discussion of Eq. (22), experiments can only probe $S_{\psi K}^{e}$ and $C_{\psi K}^{e}$, which include these terms.

- While we are not aware of any way to constrain $\hat{\theta}_{\psi K}$ from tree-level processes, it may contribute to measurable effects, such as CPT violation in mixing, at the loop level. In the absence of a rigorous framework that incorporates CPT violation, it is impossible to calculate such effects.

- It would of course be extremely exciting if the BABAR measurement is affected by CPT violating effects.

An additional interesting feature of Eqs. (36) is the appearance of terms that are quadratic in T-odd parameters,

$$
\begin{aligned}
\left.\Delta S_{T}^{+} \text {(quadratic in T-odd parameters }\right) & =-2 \hat{G}_{\psi K} \hat{S}_{\psi K} G_{\ell}^{-} \\
\left.\Delta C_{T}^{+} \text {(quadratic in T-odd parameters }\right) & =2 \hat{S}_{\psi K} S_{\ell}^{-} .
\end{aligned}
$$

While these terms would vanish if we take all T-odd parameters to zero, they are still Tconserving. Note that since we expand to linear order in all T-odd parameters, except for $\hat{S}_{\psi K_{S}}$, there are additional T conserving, $\hat{S}_{\psi K}$-independent, contributions that are quadratic in T-odd parameters that are not presented in Eqs. (38). Since $\hat{G}_{\psi K}^{2}+\hat{S}_{\psi K}^{2} \leq 1$, the maximal absolute value of the term on the right-hand side of Eq. (38) for $\Delta S_{T}^{+}$is $1,\left|2 \hat{G}_{\psi K} \hat{S}_{\psi K} G_{\ell}^{-}\right| \leq 1$. Thus, if experiments establish $\left|\Delta S_{T}^{+}\right|>1$, such a result cannot be explained by this term alone.

We are now also able to formulate the conditions under which the BABAR measurement would demonstrate $\mathrm{T}$ violation unambiguously:

$$
\hat{\theta}_{\psi K}=G_{\ell}^{-}=S_{\ell}^{-}=0
$$

In words, the necessary conditions are the following:

- The absence of CPT violation in strangeness changing decays.

- The absence of wrong sign decays or, if wrong sign decays occur, the absence of direct $\mathrm{CP}$ violation in semileptonic decays. 


\section{The time dependence of $A_{T}$}

It is often assumed that the time-dependence of the time-reversal asymmetry $A_{T}$ is of the form of Eq. (2). Eqs. (33) and (34) reveal, however, that even when taking $y=0$ and expanding to linear order in the various small parameters, the time dependence is more complicated:

$$
A_{T}=R_{T}+C_{T} \cos (x \Gamma t)+S_{T} \sin (x \Gamma t)+B_{T} \sin ^{2}(x \Gamma t)+D_{T} \sin (x \Gamma t) \cos (x \Gamma t) .
$$

Even when we neglect CPT violation, wrong sign decays, and wrong strangeness decays, the time dependence is, in general, more complicated than Eq. (2). If, in addition, one neglects $\mathrm{CP}$ violation in decay, then the only source of $\mathrm{T}$ violating effects is in mixing and should therefore vanish at $t=0$ :

$$
A_{T}=-R_{M}[1-\cos (x \Gamma t)]-\hat{S}_{\psi K} \sin (x \Gamma t)+R_{M} \hat{S}_{\psi K}^{2} \sin ^{2}(x \Gamma t)
$$

One may argue that $R_{M}$ is experimentally constrained to be very small. But then one should

also take into account the fact that $\hat{C}_{\psi K}$ is experimentally constrained to be very small, and the asymmetry has the simpler well-known form

$$
A_{T}=-\hat{S}_{\psi K} \sin (x \Gamma t)
$$

Moreover, the $\hat{S}_{\psi K}$ parameter is measured and known. The whole point of the BABAR measurement is not to measure the values of the parameters, but to demonstrate time-reversal violation in processes that are not CP-conjugate. It is perhaps more appropriate not to take experimental information from previous measurements.

\section{CP VIOLATION IN RIGHT-STRANGENESS DECAYS}

A-priori, one would expect that direct $\mathrm{CP}$ violation in right-strangeness decays is enough to allow for $A_{T} \neq 0$ even in the T-symmetry limit. However, in the previous section we found that this is not the case. In this section we first explain the reasoning behind the naive expectation, and, second, obtain the conditions under which the two processes measured by BABAR are not $\mathrm{T}$ conjugate.

In $\Gamma_{\left(\psi K_{L}\right)_{\perp}, \ell^{-} X}$, the initial $B$-meson state is orthogonal to the one that decays to $\psi K_{L}$. In $\Gamma_{\left(\ell^{+} X\right)_{\perp}, \psi K_{S}}$, the final state is the one that decays into $\psi K_{S}$. Are these two states identical? They would be if the state that does not decay to $\psi K_{L},\left|B_{\left(\rightarrow \psi K_{L}\right)_{\perp}}\right\rangle$, and the state that does not decay into $\psi K_{S},\left|B_{\left(\rightarrow \psi K_{S}\right)_{\perp}}\right\rangle$, were orthogonal to each other. Using

$$
\begin{aligned}
\left|B_{\left(\rightarrow \psi K_{L}\right)_{\perp}}^{\text {in }}\right\rangle & =N_{L}\left[\bar{A}_{\psi K_{L}}\left|B^{0}\right\rangle-A_{\psi K_{L}}\left|\bar{B}^{0}\right\rangle\right], \\
\left|B_{\left(\rightarrow \psi K_{S}\right)_{\perp}}^{\text {in }}\right\rangle & =N_{S}\left[\bar{A}_{\psi K_{S}}\left|B^{0}\right\rangle-A_{\psi K_{S}}\left|\bar{B}^{0}\right\rangle\right],
\end{aligned}
$$


where $N_{S, L}$ are normalization coefficients, and neglecting wrong strangeness decays, we obtain

$$
\left\langle B_{\left(\rightarrow \psi K_{S}\right)_{\perp}} \mid B_{\left(\rightarrow \psi K_{L}\right)_{\perp}}\right\rangle \propto \mathcal{A}_{\psi K}
$$

where $\mathcal{A}_{f}$ is the direct asymmetry defined in Eq. (15). (The same orthogonality condition can be obtained by using $\left\langle B_{\rightarrow \psi K_{S}} \mid B_{\left.\rightarrow \psi K_{L}\right)}\right\rangle$.) The conclusion is that the state that is orthogonal to the one that decays to $\psi K_{L}$ and the state that decays to $\psi K_{S}$ are not the same state, unless there is no direct $\mathrm{CP}$ violation in the $B \rightarrow \psi K$ decays. This is presumably the reason why the theoretical paper [4] and the experimental paper [1] explicitly state that they neglect direct $\mathrm{CP}$ violation.

However, as we learned from the analysis in Section III, the correct question is not whether the state that does not decay to $\psi K_{L}$ is the same as the state that decays to $\psi K_{S}$. Instead, the question is whether it is the same as the state generated in the inverse decay of $\psi K_{S}$. (The orthogonality of the two $B$-mesons at $t_{1}$ is guaranteed by the EPR entanglement, allowing one to label the initial $B$ state at $t_{1}$.) This would be the case if $\left|B_{\left(\rightarrow \psi K_{L}\right)_{\perp}}\right\rangle$ and $\left|B_{\left(\psi K_{S} \rightarrow\right)_{\perp}}\right\rangle$ were orthogonal. Using

$$
\begin{aligned}
\left|B_{\psi K_{S} \rightarrow}^{\mathrm{in}}\right\rangle & =N_{S}^{\mathrm{ID}}\left[A_{\psi K_{S}}^{\mathrm{ID}}\left|B^{0}\right\rangle+\bar{A}_{\psi K_{S}}^{\mathrm{ID}}\left|\bar{B}^{0}\right\rangle\right], \\
\left|B_{\left(\psi K_{S} \rightarrow\right)_{\perp}}^{\mathrm{in}}\right\rangle & =N_{S}^{\mathrm{ID}}\left[\bar{A}_{\psi K_{S}}^{\mathrm{ID} *}\left|B^{0}\right\rangle-A_{\psi K_{S}}^{\mathrm{ID} *}\left|\bar{B}^{0}\right\rangle\right],
\end{aligned}
$$

we obtain

$$
\begin{aligned}
\left\langle B_{\left(\psi K_{S} \rightarrow\right)_{\perp}} \mid B_{\left(\rightarrow \psi K_{L}\right)_{\perp}}\right\rangle & \propto \lambda_{\psi K_{S}}+\lambda_{\psi K_{L}}+\left(\lambda_{\psi K_{S}}-\lambda_{\psi K_{L}}\right) \hat{\theta}_{\psi K} \\
& \propto \Delta C_{\psi K}-\frac{\Delta G_{\psi K}+i \Delta S_{\psi K}}{\hat{G}_{\psi K}+i \hat{S}_{\psi K}}-\hat{\theta}_{\psi K} .
\end{aligned}
$$

Similarly,

$$
\begin{aligned}
\left\langle B_{\left(\psi K_{L} \rightarrow\right)_{\perp}} \mid B_{\left(\rightarrow \psi K_{S}\right)_{\perp}}\right\rangle & \propto \lambda_{\psi K_{L}}+\lambda_{\psi K_{S}}+\left(\lambda_{\psi K_{L}}-\lambda_{\psi K_{S}}\right) \hat{\theta}_{\psi K} \\
& \propto \Delta C_{\psi K}-\frac{\Delta G_{\psi K}+i \Delta S_{\psi K}}{\hat{G}_{\psi K}+i \hat{S}_{\psi K}}+\hat{\theta}_{\psi K} .
\end{aligned}
$$

We thus learn that the state that is orthogonal to the one that decays to $\psi K_{L}\left(\psi K_{S}\right)$ is the same as the state that is generated in the inverse decay of $\psi K_{S}\left(\psi K_{L}\right)$, unless there are wrong strangeness decays or CPT violation in strangeness changing decays. In the absence of these phenomena, the two processes are related by exchange of the initial and final CP-tagged states, as required for time-reversal conjugate processes.

One can repeat an analogous analysis for the lepton-tagged states. The question to be asked is whether the state that does not decay to $\ell^{+} X$ is orthogonal to the state that is not generated in the inverse decay of $\ell^{-} X$. For the overlap between these two states, we obtain:

$$
\left\langle B_{\left(\ell^{-} X \rightarrow\right)_{\perp}} \mid B_{\left(\rightarrow \ell^{+} X\right)_{\perp}}\right\rangle \propto \lambda_{\ell^{+}}
$$


If $\lambda_{\ell^{+}}=0$, the two states are orthogonal. We thus learn that the state that is orthogonal to the one that decays into $\ell^{+} X$ is the same as the state that is generated in the inverse decay of $\ell^{-} X$, unless there are wrong sign decays and wrong sign inverse decays. If wrong sign decays can be neglected, then the two processes are related by exchange of the initial and final lepton-tagged states, as required for time-reversal conjugate processes. (For a related asymmetry, involving $\Gamma_{\left(\psi K_{L}\right)_{\perp}, \ell^{+} X}$ and $\Gamma_{\left(\ell^{-} X\right)_{\perp}, \psi K_{S}}$, the corresponding condition is $1 / \lambda_{\ell^{-}}=0$.)

If Eq. (46) and Eq. (48) are both zero, then $\Gamma_{\left(\psi K_{L}\right)_{\perp}, \ell^{-} X}=\Gamma_{\left(\psi K_{S}\right)^{T}, \ell^{-} X}$ and $\Gamma_{\left(\ell^{+} X\right)_{\perp}, \psi K_{S}}=$ $\Gamma_{\left(\ell^{-} X\right)^{T}, \psi K_{S}}$ (under proper normalization such that the number of initial $B$ 's are equal). In this case, $A_{T}$ as defined in Eq. (1) is indeed a T-asymmetry. We conclude that if wrong strangeness decays, wrong sign decays and CPT violation in strangeness changing decays can be neglected, then the two processes measured by BABAR represent two T-conjugate processes, and then there should be no T conserving contributions to $\Delta S_{T}^{+}$and $\Delta S_{T}^{-}$. In particular, one need not assume the absence of direct $\mathrm{CP}$ violation.

\section{CONCLUSIONS}

The BABAR collaboration has measured time-reversal asymmetries in $B$ decays. Two main ingredients - the EPR effect between the two $B$-mesons produced in $\Upsilon(4 S)$ decays and the availability of both lepton-tagging and CP-tagging - allow the experimenters to approximately realize the main principle of time-reversal conjugate processes: exchanging initial and final states.

A precise exchange is impossible. The final state is identified by the $B$-meson decay, and the T-conjugate process requires, therefore, that a $B$-meson is produced in the corresponding inverse decay. Instead, the experimenters measure a process where the initial $B$-meson is identified by the decay of the other, entangled $B$-meson. We showed, however, that the initial $B$-meson prepared by lepton tagging, and the one that would be produced in the appropriate inverse decay are not identical only if there are wrong-sign decays. The initial $B$-meson prepared by $\mathrm{CP}$ tagging, and the one that would be produced in the appropriate inverse decay, are not identical only if there are wrong-strangeness contributions, or in the presence of CPT violation in decays.

The effect of CPT violation in decay has gained very little attention in the literature. One reason is that it can only be probed by measuring both decay rates and inverse decay rates, but the latter are practically inaccessible to experiments. For precisely this reason, there are no bounds on these effects. In principle, they could play a significant role in the asymmetries measured by BABAR, in spite of the fact that they are $\mathrm{T}$ conserving.

As concerns the questions posed in the Introduction, we find the following answers:

- The $A_{T}$ measurement by BABAR would vanish in the T-symmetry limit provided that $\mathrm{CPT}$ is conserved in strangeness changing decays (see Eq. (37)). 
- The initial state in each of the two processes would be the T-conjugate of the final state in the other, provided that there are neither wrong strangeness decays nor wrong sign decays nor CPT violation in strangeness changing decays. In particular, there is no need to assume the absence of direct CP violation (see Eqs. (46)-(48)).

Both wrong-sign and wrong-strangeness effects are expected to be very small. Yet, the existing experimental upper bounds on these effects are rather weak. The same set of measurements used for the time-reversal asymmetries can be used (in different combinations) to constrain also the wrong-sign and wrong-strangeness contributions.

While in this work we concentrated on very specific measurements in $B$ decays, our results are more general. They apply straightforwardly, with minor changes, to other meson systems. The main ideas also apply to neutrino oscillation experiments. Observation of $P\left(\nu_{\alpha} \rightarrow \nu_{\beta}\right) \neq P\left(\nu_{\beta} \rightarrow \nu_{\alpha}\right)$ has been advocated as a way to establish T-violation. Such a result can arise, however, also from nonstandard interactions in the production or the detection processes [21 23].

\section{Acknowledgements}

We thank José Bernabéu, Klaus Schubert and Abner Soffer for useful correspondence. EA thanks the Kupcinet-Getz Program for their hospitality at the Weizmann Institute. YG is a Weston Visiting Professor at the Weizmann Institute. This work was partially supported by a grant from the Simons Foundation (\#267432 to YG). The work of YG is supported in part by the U.S. National Science Foundation through grant PHY-0757868 and by the United States-Israel Binational Science Foundation (BSF) under grant No. 2010221. YN is the Amos de-Shalit chair of theoretical physics. YN is supported by the Israel Science Foundation, by the I-CORE program of the Planning and Budgeting Committee and the Israel Science Foundation (grant number 1937/12), and by the German-Israeli foundation for scientific research and development (GIF).

\section{Appendix A: Relevant data}

How certain is it that the small parameters listed in Eq. (32) are indeed small? In this Appendix, we compile the relevant experimental constraints. We caution the reader that some of these constraints were derived making assumptions that we do not make. For example, CPT symmetry is assumed when deriving the bounds on $R_{M}$. Thus, the upper bounds and ranges that we quote below assume no cancelations among different contributions and, even then, should be taken only as rough estimates. 
Based on Ref. [24], we find the following range for $R_{M}$ :

$$
\begin{array}{ll}
R_{M}=(-0.2 \pm 2.0) \times 10^{-3} & \text { from } \Upsilon(4 \mathrm{~S}), \\
R_{M}=(+0.3 \pm 0.9) \times 10^{-3} & \text { world average } .
\end{array}
$$

Ref. [25] uses the BABAR measurement [26] to constrain $z$ :

$$
z^{R}=(-2 \pm 5) \times 10^{-2}, \quad z^{I}=(+0.8 \pm 0.4) \times 10^{-2} .
$$

Ref. 24] obtains the following ranges for the $S_{\psi K_{S, L}}$ and $C_{\psi K_{S, L}}$ parameters:

$$
\begin{array}{ll}
S_{\psi K_{S}}=+0.665 \pm 0.024, & C_{\psi K_{S}}=+0.024 \pm 0.026 \\
S_{\psi K_{L}}=-0.663 \pm 0.041, & C_{\psi K_{L}}=-0.023 \pm 0.030 .
\end{array}
$$

Note that here we take $S_{\psi K_{L}} \rightarrow-S_{\psi K_{L}}$ with respect to [24]. Naively combining these ranges, we obtain

$$
\begin{array}{ll}
\Delta C_{\psi K}=+0.02 \pm 0.02, & \hat{C}_{\psi K}=+0.00 \pm 0.02 \\
\Delta S_{\psi K}=+0.00 \pm 0.02, & \hat{S}_{\psi K}=+0.66 \pm 0.02
\end{array}
$$

Direct bounds on wrong-strangeness $B$ decays are given by the BABAR collaboration in Ref. [27]. Ref. [25] quotes $\left|\lambda_{\psi K^{* 0}}\right|<0.25$ at $95 \%$ CL, which is weaker than the above results.

As concerns wrong-sign $B$ decays, we use the individual branching ratios from Ref. [25] to obtain

$$
\frac{\mathrm{BR}\left(B^{+} \rightarrow \ell^{+} \nu X\right)}{\mathrm{BR}\left(B^{0} \rightarrow \ell^{+} \nu X\right)}=1.06 \pm 0.04
$$

In the isospin limit and in the absence of wrong-sign decays, we should have

$$
\frac{\operatorname{BR}\left(B^{+} \rightarrow \ell^{+} \nu X\right)}{\operatorname{BR}\left(B^{0} \rightarrow \ell^{+} \nu X\right)}=\frac{\tau_{B^{+}}}{\tau_{B^{0}}}=1.08 \pm 0.01 .
$$

Comparing Eq. (A7) to (A8) we obtain, at $2 \sigma$

$$
\left|\bar{A}_{\ell^{+}} / A_{\ell^{+}}\right|<0.44 \text {. }
$$

Using the exclusive process $B^{+} \rightarrow \ell^{+} \nu \bar{D}^{0}$ instead of the inclusive one results in a weaker bound. One can also use different tagging techniques in measurements of CP asymmetries to place bounds on wrong sign decays. Assuming CPT to be a good symmetry, we find

$$
C_{C P}^{\text {lep }}-C_{C P}^{\mathrm{Kaon}}=\hat{S}_{\psi K_{S}} S_{\ell}^{+},
$$

where $C_{C P}^{\text {lep }}$ and $C_{C P}^{\text {Kaon }}$ are the measured coefficients for the $\cos (x \Gamma t)$ function with lepton and Kaon tagging, respectively. Using the results of [28] and Eq. (A6) we get, at 2 $\sigma$,

$$
\left|S_{\ell}^{+}\right|<0.24
$$




\section{Appendix B: Isolating parameters of interest}

Combinations of observables measured by $B A B A R$ allow us to constrain various parameters

of interest. We here use BABAR 's notation. The combinations that correspond to the CPodd $C_{\psi K}^{e}$ and $S_{\psi K}^{e}$, defined in Eq. (22), can be isolated via the following combinations:

$$
\begin{aligned}
-\frac{1}{2}\left(C_{\ell^{+}, K_{S}}^{ \pm}+C_{\ell^{+}, K_{L}}^{ \pm}\right) & =\frac{1}{2}\left(C_{\ell^{-}, K_{S}}^{ \pm}+C_{\ell^{-}, K_{L}}^{ \pm}\right)=\hat{C}_{\psi K}+\hat{\theta}_{\psi K}^{R}, \\
\frac{ \pm 1}{2}\left(S_{\ell^{+}, K_{S}}^{ \pm}-S_{\ell^{+}, K_{L}}^{ \pm}\right) & =\frac{\mp 1}{2}\left(S_{\ell^{-}, K_{S}}^{ \pm}-S_{\ell^{-}, K_{L}}^{ \pm}\right)=\hat{S}_{\psi K}-\hat{G}_{\psi K} \hat{\theta}_{\psi K}^{I} .
\end{aligned}
$$

Upper bounds on combinations of the wrong-sign T-odd parameters $S_{\ell}^{-}$and $G_{\ell}^{-}$, defined in Eq. (19), and the CPT violating parameter $z$, defined in Eq. (7), can be obtained as follows:

$$
\begin{aligned}
& \frac{-1}{2}\left(C_{\ell^{+}, K_{S}}^{ \pm}+C_{\ell^{-}, K_{S}}^{ \pm}\right)=S_{\psi K_{S}}\left(S_{\ell}^{-}-z^{I}\right), \\
& \frac{-1}{2}\left(C_{\ell^{+}, K_{L}}^{ \pm}+C_{\ell^{-}, K_{L}}^{ \pm}\right)=S_{\psi K_{L}}\left(S_{\ell}^{-}-z^{I}\right), \\
& \frac{ \pm 1}{2}\left(S_{\ell^{+}, K_{S}}^{ \pm}+S_{\ell^{-}, K_{S}}^{ \pm}\right)=G_{\psi K_{S}} S_{\psi K_{S}}\left(G_{\ell}^{-}-z^{R}\right), \\
& \frac{ \pm 1}{2}\left(S_{\ell^{+}, K_{L}}^{ \pm}+S_{\ell^{-}, K_{L}}^{ \pm}\right)=G_{\psi K_{L}} S_{\psi K_{L}}\left(G_{\ell}^{-}-z^{R}\right),
\end{aligned}
$$

From the results of [1] we can get

$$
\hat{S}_{\psi K}-\hat{G}_{\psi K} \hat{\theta}_{\psi K}^{I}=0.69 \pm 0.04
$$

and the following bounds can be deduced

$$
\begin{aligned}
& \left|\hat{C}_{\psi K}+\hat{\theta}_{\psi K}^{R}\right|<0.07 \\
& \left|G_{\psi K_{S, L}} S_{\psi K_{S, L}}\left(G_{\ell}^{-}-z^{R}\right)\right|<0.10 \\
& \left|S_{\psi K_{S, L}}\left(S_{\ell}^{-}-z^{I}\right)\right|<0.06
\end{aligned}
$$

at $2 \sigma$ level. In case we assume no CPT violation naive combination of the above will lead to

$$
\left|S_{\ell}^{-}\right|<0.10, \quad\left|G_{\ell}^{-}\right|<0.21
$$

at $2 \sigma$ level.

\section{Appendix C: Experimental asymmetries}

In this Appendix, we provide the full expressions for the asymmetries measured at $B A B A R$. For the relation between our notation (30) and those of Table I of [1], see Eq. 
(35) and the discussion below it. The asymmetries measured by BABAR are the following:

$$
\begin{gathered}
\Delta S_{T}^{+}=\frac{S_{\left(\psi K_{L}\right)_{\perp}, \ell^{-} X}}{\kappa_{\left(\psi K_{L}\right)_{\perp}, \ell^{-} X}}-\frac{S_{\left(\ell^{+} X\right)_{\perp}, \psi K_{S}}}{\kappa_{\left(\ell^{+} X\right)_{\perp}, \psi K_{S}}}, \quad \Delta C_{T}^{+}=\frac{C_{\left(\psi K_{L}\right)_{\perp}, \ell^{-} X}}{\kappa_{\left(\psi K_{L}\right)_{\perp}, \ell^{-} X}}-\frac{C_{\left(\ell^{+} X\right)_{\perp}, \psi K_{S}}}{\kappa_{\left(\ell^{+} X\right)_{\perp}, \psi K_{S}}}, \\
\Delta S_{T}^{-}=\frac{S_{\left(\ell^{-} X\right)_{\perp}, \psi K_{L}}}{\kappa_{\left(\ell^{-} X\right)_{\perp}, \psi K_{L}}}-\frac{S_{\left(\psi K_{S}\right)_{\perp}, \ell^{+} X}}{\kappa_{\left(\psi K_{S}\right)_{\perp}, \ell^{+} X}}, \quad \Delta C_{T}^{-}=\frac{C_{\left(\ell^{-} X\right)_{\perp}, \psi K_{L}}}{\kappa_{\left(\ell^{-} X\right)_{\perp}, \psi K_{L}}}-\frac{C_{\left(\psi K_{S}\right)_{\perp}, \ell^{+} X}}{\kappa_{\left(\psi K_{S}\right)_{\perp}, \ell^{+} X}}, \\
\Delta S_{C P}^{+}=\frac{S_{\left(\ell^{-} X\right)_{\perp}, \psi K_{S}}}{\kappa_{\left(\ell^{-} X\right)_{\perp}, \psi K_{S}}}-\frac{S_{\left(\ell^{+} X\right)_{\perp}, \psi K_{S}}}{\kappa_{\left(\ell^{+} X\right)_{\perp}, \psi K_{S}}}, \quad \Delta C_{C P}^{+}=\frac{C_{\left(\ell^{-} X\right)_{\perp}, \psi K_{S}}}{\kappa_{\left(\ell^{-} X\right)_{\perp}, \psi K_{S}}}-\frac{C_{\left(\ell^{+} X\right)_{\perp}, \psi K_{S}}}{\kappa_{\left(\ell^{+} X\right)_{\perp}, \psi K_{S}}}, \\
\Delta S_{C P}^{-}=\frac{S_{\left(\psi K_{S}\right)_{\perp}, \ell^{-} X}}{\kappa_{\left(\psi K_{S}\right)_{\perp}, \ell^{-} X}}-\frac{S_{\left(\psi K_{S}\right)_{\perp}, \ell^{+} X}}{\kappa_{\left(\psi K_{S}\right)_{\perp}, \ell^{+} X}}, \quad \Delta C_{C P}^{-}=\frac{C_{\left(\psi K_{S}\right)_{\perp}, \ell^{-} X}}{\kappa_{\left(\psi K_{S}\right)_{\perp}, \ell^{-} X}}-\frac{C_{\left(\psi K_{S}\right)_{\perp}, \ell^{+} X}}{\kappa_{\left(\psi K_{S}\right)_{\perp}, \ell^{+} X}}, \\
\Delta S_{C P T}^{+}=\frac{S_{\left(\psi K_{L}\right)_{\perp}, \ell^{+} X}}{\kappa_{\left(\psi K_{L}\right)_{\perp}, \ell^{+} X}}-\frac{S_{\left(\ell^{+} X\right)_{\perp}, \psi K_{S}}}{\kappa_{\left(\ell^{+} X\right)_{\perp}, \psi K_{S}}}, \quad \Delta C_{C P T}^{+}=\frac{C_{\left(\psi K_{L}\right)_{\perp}, \ell^{+} X}}{\kappa_{\left(\psi K_{L}\right)_{\perp}, \ell^{+} X}}-\frac{C_{\left(\ell^{+} X\right)_{\perp}, \psi K_{S}}}{\kappa_{\left(\ell^{+} X\right)_{\perp}, \psi K_{S}}}, \\
\Delta S_{C P T}^{-}=\frac{S_{\left(\ell^{+} X\right)_{\perp}, \psi K_{L}}}{\kappa_{\left(\ell^{+} X\right)_{\perp}, \psi K_{L}}}-\frac{S_{\left(\psi K_{S}\right)_{\perp}, \ell^{+} X}}{\kappa_{\left(\psi K_{S}\right)_{\perp}, \ell^{+} X}}, \quad \Delta C_{C P T}^{-}=\frac{C_{\left(\ell^{+} X\right)_{\perp}, \psi K_{L}}}{\kappa_{\left(\ell^{+} X\right)_{\perp}, \psi K_{L}}}-\frac{C_{\left(\psi K_{S}\right)_{\perp}, \ell^{+} X}}{\kappa_{\left(\psi K_{S}\right)_{\perp}, \ell^{+} X}} .
\end{gathered}
$$

We find the following expressions for these asymmetries:

$$
\begin{aligned}
\Delta S_{T}^{+} & =-\Delta S_{T}^{-}=-2\left[\hat{S}_{\psi K}\left(1+\hat{G}_{\psi K}\left(G_{\ell}^{-}-z^{R}\right)\right)-\hat{G}_{\psi K} \hat{\theta}_{\psi K}^{I}\right], \\
\Delta C_{T}^{+} & =\Delta C_{T}^{-}=2\left[\hat{C}_{\psi K}+\hat{S}_{\psi K}\left(S_{\ell}^{-}-z^{I}\right)+\hat{\theta}_{\psi K}^{R}\right], \\
\Delta S_{C P}^{+} & =-\Delta S_{C P}^{-}=-2\left[S_{\psi K_{S}}-G_{\psi K_{S}} \theta_{\psi K_{S}}^{I}+S_{\psi K_{S}} G_{\psi K_{S}} G_{\ell}^{+}-z^{I}\left(1-\hat{S}_{\psi K}^{2}\right)\right], \\
\Delta C_{C P}^{+} & =\Delta C_{C P}^{-}=2\left[C_{\psi K_{S}}+S_{\psi K_{S}} S_{\ell}^{+}+\theta_{\psi K_{S}}^{R}+G_{\psi K_{S}} z^{R}\right], \\
\Delta S_{C P T}^{+} & =-\Delta S_{C P T}^{-}=-2\left[\Delta S_{\psi K}-z^{I}\left(1-\hat{S}_{\psi K}^{2}\right)+\hat{G}_{\psi K}\left(\hat{S}_{\psi K} G_{\ell^{+}}-\Delta \theta_{\psi K}^{I}-\hat{S}_{\psi K} z^{R}\right)\right], \\
\Delta C_{C P T}^{+} & =\Delta C_{C P T}^{-}=2\left[\Delta C_{\psi K}+\Delta \theta_{\psi K}^{R}+\hat{S}_{\psi K}\left(S_{\ell^{+}}-z^{I}\right)+\hat{G}_{\psi K} z^{R}\right] .
\end{aligned}
$$

We notice that not only do the T-asymmetries get T-even contributions, as explained in Section IIIB, but also the CPT asymmetries get CPT-even contributions. All of these

effects vanish if there is neither CPT violation in strangeness changing decays nor wrong strangeness decays nor wrong sign decays.

\section{Appendix D: Theoretical asymmetries}

It is interesting to define "theoretical" asymmetries where the initial states of the corresponding experimental asymmetries are replaced by the time-conjugate of the final states. Thus, instead of the experimental method, of observing one of two entangled $B$-mesons decaying, thus projecting the other $B$-meson onto the orthogonal state, here we refer to 
corresponding "gedanken experiments", that start with inverse decays:

$$
\begin{gathered}
\Delta S_{T}^{+(t)}=\frac{S_{\left(\psi K_{S}\right)^{T}, \ell^{-} X}}{\kappa_{\left(\psi K_{S}\right)^{T}, \ell^{-} X}}-\frac{S_{\left(\ell^{-} X\right)^{T}, \psi K_{S}}}{\kappa_{\left(\ell^{-} X\right)^{T}, \psi K_{S}}}, \quad \Delta C_{T}^{+(t)}=\frac{C_{\left(\psi K_{S}\right)^{T}, \ell^{-} X}}{\kappa_{\left(\psi K_{S}\right)^{T}, \ell^{-} X}}-\frac{C_{\left(\ell^{-} X\right)^{T}, \psi K_{S}}}{\kappa_{\left(\ell^{-} X\right)^{T}, \psi K_{S}}}, \\
\Delta S_{T}^{-(t)}=\frac{S_{\left(\ell^{+} X\right)^{T}, \psi K_{L}}}{\kappa_{\left(\ell^{+} X\right)^{T}, \psi K_{L}}}-\frac{S_{\left(\psi K_{L}\right)^{T}, \ell^{+} X}}{\kappa_{\left(\psi K_{L}\right)^{T}, \ell^{+} X}}, \quad \Delta C_{T}^{-(t)}=\frac{C_{\left(\ell^{+} X\right)^{T}, \psi K_{L}}}{\kappa_{\left(\ell^{+} X\right)^{T}, \psi K_{L}}}-\frac{C_{\left(\psi K_{L}\right)^{T}, \ell^{+} X}}{\kappa_{\left(\psi K_{L}\right)^{T}, \ell^{+} X}}, \\
\Delta S_{C P}^{+(t)}=\frac{S_{\left(\ell^{+} X\right)^{T}, \psi K_{S}}}{\kappa_{\left(\ell^{+} X\right)^{T}, \psi K_{S}}}-\frac{S_{\left(\ell^{-} X\right)^{T}, \psi K_{S}}}{\kappa_{\left(\ell^{-} X\right)^{T}, \psi K_{S}}}, \quad \Delta C_{C P}^{+(t)}=\frac{C_{\left(\ell^{+} X\right)^{T}, \psi K_{S}}}{\kappa_{\left(\ell^{+} X\right)^{T}, \psi K_{S}}}-\frac{C_{\left(\ell^{-} X\right)^{T}, \psi K_{S}}}{\kappa_{\left(\ell^{-} X\right)^{T}, \psi K_{S}}}, \\
\Delta S_{C P}^{-(t)}=\frac{S_{\left(\psi K_{L}\right)^{T}, \ell^{-} X}}{\kappa_{\left(\psi K_{L}\right)^{T}, \ell^{-} X}}-\frac{S_{\left(\psi K_{L}\right)^{T}, \ell^{+} X}}{\kappa_{\left(\psi K_{L}\right)^{T}, \ell^{+} X}}, \quad \Delta C_{C P}^{-(t)}=\frac{C_{\left(\psi K_{L}\right)^{T}, \ell^{-} X}}{\kappa_{\left(\psi K_{L}\right)^{T}, \ell^{-} X}}-\frac{C_{\left(\psi K_{L}\right)^{T}, \ell^{+} X}}{\kappa_{\left(\psi K_{L}\right)^{T}, \ell^{+} X}}, \\
\Delta S_{C P T}^{+(t)}=\frac{S_{\left(\psi K_{S}\right)^{T}, \ell^{+} X}}{\kappa_{\left(\psi K_{S}\right)^{T}, \ell^{+} X}}-\frac{S_{\left(\ell^{-} X\right)^{T}, \psi K_{S}}}{\kappa_{\left(\ell^{-} X\right)^{T}, \psi K_{S}}}, \quad \Delta C_{C P T}^{+(t)}=\frac{C_{\left(\psi K_{S}\right)^{T}, \ell^{+} X}}{\kappa_{\left(\psi K_{S}\right)^{T}, \ell^{+} X}}-\frac{C_{\left(\ell^{-} X\right)^{T}, \psi K_{S}}}{\kappa_{\left(\ell^{-} X\right)^{T}, \psi K_{S}}}, \\
\Delta S_{C P T}^{-(t)}=\frac{S_{\left(\ell^{-} X\right)^{T}, \psi K_{L}}}{\kappa_{\left(\ell^{-} X\right)^{T}, \psi K_{L}}}-\frac{S_{\left(\psi K_{L}\right)^{T}, \ell^{+} X}}{\kappa_{\left(\psi K_{L}\right)^{T}, \ell^{+} X}}, \quad \Delta C_{C P T}^{-(t)}=\frac{C_{\left(\ell^{-} X\right)^{T}, \psi K_{L}}}{\kappa_{\left(\ell^{-} X\right)^{T}, \psi K_{L}}}-\frac{C_{\left(\psi K_{L}\right)^{T}, \ell^{+} X}}{\kappa_{\left(\psi K_{L}\right)^{T}, \ell^{+} X}} .
\end{gathered}
$$

We use the same approximations as in Sec. III. We find:

$$
\begin{aligned}
\Delta S_{T}^{+(t)} & =-2 S_{\psi K_{S}}\left[1-G_{\psi K_{S}}\left(z^{R}+G_{\ell}^{+}\right)\right] \\
\Delta S_{T}^{-(t)} & =-2 S_{\psi K_{L}}\left[1+G_{\psi K_{L}}\left(z^{R}-G_{\ell}^{+}\right)\right] \\
\Delta C_{T}^{+(t)} & =2\left[C_{\psi K_{S}}-S_{\psi K_{S}}\left(z^{I}+S_{\ell}^{+}\right)\right] \\
\Delta C_{T}^{-(t)} & =2\left[C_{\psi K_{L}}+S_{\psi K_{L}}\left(z^{I}-S_{\ell}^{+}\right)\right] \\
\Delta S_{C P}^{+(t)} & =-2\left[S_{\psi K_{S}}-G_{\psi K_{S}} \theta_{\psi K_{S}}^{I}-S_{\psi K_{S}} G_{\psi K_{S}} G_{\ell}^{+}-z^{I}\left(1-S_{\psi K_{S}}^{2}\right)\right], \\
\Delta S_{C P}^{-(t)} & =-2\left[S_{\psi K_{L}}+G_{\psi K_{L}} \theta_{\psi K_{L}}^{I}-S_{\psi K_{L}} G_{\psi K_{L}} G_{\ell}^{+}+z^{I}\left(1-S_{\psi K_{L}}^{2}\right)\right], \\
\Delta C_{C P}^{+(t)} & =2\left[C_{\psi K_{S}}-S_{\psi K_{S}} S_{\ell}^{+}+\theta_{\psi K_{S}}^{R}+G_{\psi K_{S}} z^{R}\right] \\
\Delta C_{C P}^{-(t)} & =2\left[C_{\psi K_{L}}-S_{\psi K_{L}} S_{\ell}^{-}-\theta_{\psi K_{L}}^{R}-G_{\psi K_{L}} z^{R}\right] \\
\Delta S_{C P T}^{+(t)} & =2\left[z^{I}\left(1-S_{\psi K_{S}}^{2}\right)+G_{\psi K_{S}}\left(\theta_{\psi K_{S}}^{I}+S_{\psi K_{S}} z^{R}\right)\right] \\
\Delta S_{C P T}^{-(t)} & =-2\left[z^{I}\left(1-S_{\psi K_{L}}^{2}\right)+G_{\psi K_{L}}\left(\theta_{\psi K_{L}}^{I}+S_{\psi K_{L}} z^{R}\right)\right] \\
\Delta C_{C P T}^{+(t)} & =2\left[\theta_{\psi K_{S}}^{R}-S_{\psi K_{S}} z^{I}+G_{\psi K_{S}} z^{R}\right] \\
\Delta C_{C P T}^{-(t)} & =-2\left[\theta_{\psi K_{L}}^{R}-S_{\psi K_{L}} z^{I}+G_{\psi K_{L}} z^{R}\right] .
\end{aligned}
$$

As expected, the theoretical $\mathrm{T}$ asymmetries have only T-odd contributions, the theoretical $\mathrm{CP}$ asymmetries have only $\mathrm{CP}$-odd contributions, and the theoretical CPT asymmetries have only CPT-odd contributions. Furthermore, in the absence of wrong strangeness decays, wrong sign decays and CPT violation in strangeness changing decays, the theoretical asymmetries equal the corresponding experimental asymmetries.

\section{Appendix E: EPR entanglement with CPT violation}

In this appendix we show that the factorization of the decay amplitudes to $e^{-\Gamma\left(t_{1}+t_{2}\right)} \times$ $f\left(t_{2}-t_{1}\right)$ holds in the presence of CPT violation. We follow Ch. 9 of [13] with the appropriate 
modifications for the CPT violating case. The initial pair is in a state

$$
\left|\Phi^{c}\right\rangle=\frac{1}{\sqrt{2}}[|B(\vec{k})\rangle \otimes|\bar{B}(-\vec{k})\rangle-|\bar{B}(\vec{k})\rangle \otimes|B(-\vec{k})\rangle]
$$

where the relative (-) sign is a result of the C-parity of the $\Upsilon(4 S)$. The $\pm \vec{k}$ are the three momenta of the left and right moving meson in the resonance rest frame.

The decay amplitude of the meson with momenta $\vec{k}$ into final state $f_{1}$ and of the one with momenta $-\vec{k}$ to final state $f_{2}$ at times $t_{1}$ and $t_{2}$, respectively, is $\left(t \equiv t_{2}-t_{1}\right)$

$$
\begin{aligned}
\left\langle f_{1}, t_{1} ; f_{2}, t_{2}|T| \Phi^{c}\right\rangle= & \frac{e^{-(\Gamma / 2+i m)\left(t_{1}+t_{2}\right)}}{\sqrt{2}} \times \\
& {\left[\left(\sqrt{1-z^{2}}\left(\frac{p}{q} A_{f_{1}} A_{f_{2}}-\frac{q}{p} \bar{A}_{f_{1}} \bar{A}_{f_{2}}\right)+z\left(\bar{A}_{f_{2}} A_{f_{1}}+\bar{A}_{f_{1}} A_{f_{2}}\right)\right) g_{-}(t)\right.} \\
& \left.+\left(\bar{A}_{f_{2}} A_{f_{1}}-\bar{A}_{f_{1}} A_{f_{2}}\right) g_{+}(t)\right]
\end{aligned}
$$

where

$$
\begin{aligned}
& g_{+}(t)=\cos \left(\frac{x \Gamma t}{2}\right) \cosh \left(\frac{y \Gamma t}{2}\right)+i \sin \left(\frac{x \Gamma t}{2}\right) \sinh \left(\frac{y \Gamma t}{2}\right), \\
& g_{-}(t)=\cos \left(\frac{x \Gamma t}{2}\right) \sinh \left(\frac{y \Gamma t}{2}\right)+i \sin \left(\frac{x \Gamma t}{2}\right) \cosh \left(\frac{y \Gamma t}{2}\right) .
\end{aligned}
$$

By squaring the absolute value of the amplitude in Eq. (E2) we get that it factorizes as $e^{-\Gamma\left(t_{1}+t_{2}\right)} \times f(t)$.

[1] J. P. Lees et al. [BaBar Collaboration], Phys. Rev. Lett. 109, 211801 (2012) arXiv:1207.5832 [hep-ex]].

[2] M. C. Banuls and J. Bernabeu, Phys. Lett. B 464, 117 (1999) hep-ph/9908353]; M. C. Banuls and J. Bernabeu, Nucl. Phys. B 590, 19 (2000) hep-ph/0005323.

[3] L. Wolfenstein, Int. J. Mod. Phys. E 8, 501 (1999).

[4] J. Bernabeu, F. Martinez-Vidal and P. Villanueva-Perez, JHEP 1208, 064 (2012) arXiv:1203.0171 [hep-ph]].

[5] E. Alvarez and A. Szynkman, Mod. Phys. Lett. A 23, 2085 (2008) hep-ph/0611370.

[6] J. Bernabeu, F. J. Botella and M. Nebot, arXiv:1309.0439 [hep-ph]].

[7] A. Angelopoulos et al. [CPLEAR Collaboration], Phys. Lett. B 444, 43 (1998).

[8] P. K. Kabir, Phys. Rev. D 2, 540 (1970).

[9] K. R. Schubert, B. Wolff, J. C. Chollet, J. M. Gaillard, M. R. Jane, T. J. Ratcliffe and J. P. Repellin, Phys. Lett. B 31, 662 (1970).

[10] L. Lavoura, Annals Phys. 207, 428 (1991). 
[11] L. Alvarez-Gaume, C. Kounnas, S. Lola and P. Pavlopoulos, Phys. Lett. B 458, 347 (1999) hep-ph/9812326.

[12] J. P. Silva, Phys. Rev. D 62, 116008 (2000) hep-ph/0007075.

[13] G. C. Branco, L. Lavoura and J. P. Silva, Int. Ser. Monogr. Phys. 103, 1 (1999).

[14] Y. Grossman, A. L. Kagan and Z. Ligeti, Phys. Lett. B 538, 327 (2002) hep-ph/0204212.

[15] Y. I. Azimov and A. A. Iogansen, Yad. Fiz. 33, 388 (1981) [Sov. J. Nucl. Phys. 33, 205 (1981)].

[16] Y. I. Azimov, Eur. Phys. J. A 4, 21 (1999) hep-ph/9808386.

[17] H. J. Lipkin, Z. -z. Xing, Phys. Lett. B450, 405-411 (1999). hep-ph/9901329].

[18] I. I. Bigi, A. I. Sanda, Phys. Lett. B625, 47-52 (2005). hep-ph/0506037.

[19] G. Calderon, D. Delepine, G. L. Castro, Phys. Rev. D75, 076001 (2007). hep-ph/0702282.

[20] Y. Grossman and Y. Nir, JHEP 1204, 002 (2012) [arXiv:1110.3790 [hep-ph]].

[21] Y. Grossman, Phys. Lett. B 359, 141 (1995) hep-ph/9507344.

[22] M. C. Gonzalez-Garcia, Y. Grossman, A. Gusso and Y. Nir, Phys. Rev. D 64, 096006 (2001) hep-ph/0105159.

[23] B. Bellazzini, Y. Grossman, I. Nachshon and P. Paradisi, JHEP 1106, 104 (2011) arXiv:1012.3759 [hep-ph]].

[24] Y. Amhis et al. [Heavy Flavor Averaging Group Collaboration], arXiv:1207.1158 [hep-ex]].

[25] J. Beringer et al. [Particle Data Group Collaboration], Phys. Rev. D 86, 010001 (2012).

[26] B. Aubert et al. [BaBar Collaboration], Phys. Rev. D 70, 012007 (2004) hep-ex/0403002.

[27] B. Aubert et al. [BaBar Collaboration], Phys. Rev. Lett. 93, 081801 (2004) hep-ex/0404005].

[28] B. Aubert et al. [BaBar Collaboration], Phys. Rev. D 79, 072009 (2009) arXiv:0902.1708 [hep-ex]]. 